\title{
Du gouvernement royal des Hauts plateaux à l'État colonial français. L'émergence de dispositifs de polices à Madagascar
}

Nicolas Courtin

\section{OpenEdition}

Journals

Édition électronique

URL : https://journals.openedition.org/chs/1290

DOI : $10.4000 /$ chs. 1290

ISSN : 1663-4837

Éditeur

Librairie Droz

Édition imprimée

Date de publication : 1 décembre 2011

Pagination : 77-95

ISSN : $1422-0857$

Référence électronique

Nicolas Courtin, «Du gouvernement royal des Hauts plateaux à l'État colonial français. L'émergence de dispositifs de polices à Madagascar », Crime, Histoire \& Sociétés / Crime, History \& Societies [En ligne], Vol. 15, n² | 2011, mis en ligne le 01 décembre 2014, consulté le 22 mars 2022. URL : http:// journals.openedition.org/chs/1290; DOI : https://doi.org/10.4000/chs.1290 


\title{
Du gouvernement royal des Hauts plateaux à l'État colonial français
} L'émergence de dispositifs de polices à Madagascar

\author{
Nicolas Courtin*
}

\begin{abstract}
The purpose of this article is to trace the slow formation of institutional arrangements around the Madagascar police force, and to study their role in the development of the Merina Highland State (1878-1895) and the emergence from 1895 onwards of the "unity State" under French colonial rule. The article also aims at throwing light on the continuities of some of these arrangements in the transition from kingdom to colony. Finally, by historically analysing the establishment of law enforcement agencies and penal institutions in Madagascar, this research seeks to clarify the relationship between conventional order maintenance, and maintenance of the "order" that constituted the matrix of the State, and which transcended any break-down in the ties between the dominant forces of the island.
\end{abstract}

L'objet de ce travail est de retracer la lente structuration institutionnelle des dispositifs de polices à Madagascar et d'étudier leur rôle dans l'élaboration de l'Etat royal des Hauts plateaux (1878-1895) et l'émergence de l' «État complet» colonial français à partir de 1895. Il a pour but d'éclairer de même les continuités de certains de ces dispositifs entre les deux moments, royal et colonial. À travers une analyse historique de la mise en place des institutions policières et pénales à Madagascar, cette recherche tente enfin d'expliciter le rapport entre le maintien de l'ordre proprement dit et le maintien de l'Ordre comme matrice de l'État, transcendant les ruptures conjoncturelles d'autorités dominantes.

«Le maintien de la sécurité locale est la fonction première de toute administration civile ou militaire ${ }^{1}$.

Soumis à une présence et à une influence politique, militaire, religieuse et Séconomique anglaise et française ${ }^{2}$ grandissante tout au long du XIX ${ }^{\mathrm{e}}$ siècle, et malgré des remous conjoncturels, le «Royaume de Madagascar ${ }^{3}$, dont le cœur est situé sur les Hauts plateaux centraux, adopte progressivement des modalités de gouvernement issues d'expériences historiques occidentales. Le gouvernement despopulations par le droit s'incarne dans la promulgation de textes législatifs

* Actuellement secrétaire de rédaction de la revue Afrique contemporaine (Agence Française de Développement), il poursuit le Master 2 Recherche «Analyse des conflits et de la violence» à l'université de Versailles Saint-Quentin. Membre du groupe informel Gempa (Groupe d'études sur les mondes policiers africains).

1 Archives nationales d'outre mer (ANOM), 6(12)D5, Instruction $\mathrm{n}^{\circ} 12$ : Circulaire du $1^{\text {er }}$ bureau de l'État-Major du Commandant Supérieur des Troupes du Groupe de l'Afrique orientale, signée par le Général Winckel-Mayer, 16 octobre 1911.

2 Jacob (1996).

3 Esoavelomandroso (1979). 
successifs ${ }^{4}$ par les souverains malgaches des Hauts plateaux et modifie l'administration du politique dans la deuxième moitié du XIX ${ }^{\mathrm{e}}$ siècle.

Nonobstant des formes anciennes de contrôle et de régulation des populations, telle les institutions des Vadintany, «les époux de la terre», et des Andriambaventy ${ }^{5}$, l'objet de ce travail est d'éclairer les dispositifs de polices mis en place par le Premier ministre Rainilaiarivony ${ }^{6}$ lors des grandes réformes de l'État royal de la fin des années 1860 jusqu'au début des années 1880. Il s'attache à préciser, quels rôles jouent les dispositifs de polices dans la formation de l'État royal malgache et dans l'émergence d'un «État complet » colonial français à Madagascar.

Non exhaustive, cette étude n'a pour ambition ni d'embrasser l'ensemble des forces dites d'ordre, ni d'étudier en détail les pratiques policières en situation coloniale ${ }^{8}$. Elle passe par exemple sous silence le rôle premier de maintien et d'imposition de l'ordre joué par le corps d'occupation et les régiments de tirailleurs sénégalais, mixtes et malgaches dans les grands centres, les territoires militaires et les cercles ${ }^{9}$. En outre, elle se focalise davantage sur l'émergence et la construction lente des grandes structures institutionnelles policières naissantes dans les centres urbains, comme le bureau de la Sûreté à Tananarive et la police urbaine, administrative et judiciaire; et dans les provinces civiles, le rôle de police dévolu à l'administration coloniale, aux chefs de province, aux chefs de district et à la Garde indigène. Enfin, elle propose de scruter la dichotomie entre le maintien de l'ordre proprement dit et le maintien de l'Ordre comme matrice de l'émergence de l'État royal et de l'État colonial.

\section{LES DISPOSITIFS DE POLICES DANS LE «ROYAUME DE MADAGASCAR», 1878-1895}

\section{"La plus louable tentative» ${ }^{10}$ de surveillance des populations}

Au cours d'un grand kabary $^{11}$ tenu le 14 juillet 1878, le Premier ministre Rainilaiarivony annonce la création des Sakaizam-bohitra ${ }^{12}$, les «Amis des villages».

4 Vidal (1968).

5 Institués sous le règne décisif de Andrianampoinimerina (1787-1810), les Vadintany sont des agents royaux envoyés dans les territoires nouvellement conquis pour instruire les affaires judiciaires soumises au roi. Les Adriambaventy sont des magistrats de hauts rangs institués par Radama $1^{\mathrm{er}}$ (18101828). Voir Rakoto (2009).

6 Chapus (1953).

7 Michel (1994). La construction de l'État colonial est la question majeure du proconsulat de Gallieni. La notion d' «État complet» forgée par Marc Michel recouvre les volontés antagonistes de Gallieni: à la fois une volonté de «modernité » en rupture avec le passé mais, également une volonté de continuité avec les «structures traditionnelles» de la société malgache.

8 Ce dernier point fait l'objet d'un travail en cours.

9 Fremigacci (1987).

10 ANOM, 2 Z 27, «L'Institution des Sakaizam-bohitra ou Amis des villages, 14 juillet 1878 ». Préface de G. Julien du 31 décembre 1899. G. Julien, administrateur adjoint de $1^{\text {ère }}$ classe, est l'interprète principal du gouvernement général, l'un des meilleurs malgachisants de son temps et le traducteur de ce kabary.

11 Provenant de l'arabe «kabar», le «kabary» est un discours prononcé à haute voix devant un public, et dans ce contexte une forme traditionnelle de gouvernement oral des hommes. Ces dits du pouvoir royal, émanant de la personne sacrée de la reine, et ici du Premier ministre, ont force de loi.

12 ANOM, 2 Z 27, idem. 
Présents uniquement dans le cœur du «royaume de Madagascar», en Imerina (la région centrale des Hauts plateaux sous l'autorité de la royauté merina), les Sakaizam-bohitra «surveillent et renseignent plus haut et transmettent les directives vers le bas $»^{13}$. Dans le but de surveiller au plus près les populations, ils sont les yeux et les oreilles du Premier ministre dans le royaume. Cette institution administrative supplémentaire se superpose aux pouvoirs anciens en contrebalaçant leur influence et leur autorité et en accaparant les pouvoirs qu'ils possédaient. Les «autorités traditionnelles », chefs de village, ray'mandreny (les «pères et mères », les notables) et tompomenakely (les seigneurs féodaux) côtoient désormais les intermédiaires directs du Premier ministre ${ }^{14}$. La création du corps des Sakaizam-bohitra n'est donc pas en premier lieu une volonté de mettre en place une sorte d'institution policière à des fins de police mais bien l'affirmation d'un pouvoir oligarchique, autoritaire et singulier pris dans le jeu conflictuel des rapports de pouvoir entre castes et «autorités traditionnelles» en Imerina. Toutefois, cette institution incarne «le poids de l'État sous la forme de son bras militaire $»^{15}$.

L'examen des 87 articles de ce code civil et pénal, retranscription écrite du kabary, montre une volonté de centralisation de toutes les affaires publiques entre les mains du Premier ministre, l'exercice d'une surveillance étroite des sujets et la ruine de toutes les influences autres que celles autorisées à parler en son nom. "C'est le triomphe de la caste hova sur la noblesse et la royauté ${ }^{16}$, écrit Julien dans la préface de sa traduction du kabary. L'historienne Françoise Raison-Jourde illustre la perception des administrés vis-à-vis de ce nouveau corps: «il n'est que de comparer le terme lénifiant de sakaizam-bohitra (amis des campagnes), officiellement adopté, avec celui de miara-milambohitra (militaires des campagnes) employé par leurs administrés, pour enregistrer les différences de perceptions $\gg^{17}$.

En effet, plus de 6500 officiers et anciens soldats royaux à la retraite, «de la caste la plus humble $»^{18}$ du Premier ministre, «vieillis dans le métier et incapables d'un service actif ${ }^{19}$, sont répartis dans 194 chefs-lieux. Pour eux, c'est une forme de compensation pour service rendu lors de des phases successives d'expansion de la royauté ${ }^{20}$. Présents en milieu rural, «les officiers et les soldats spéciaux qui ont la garde de la ville reçoivent aussi le titre de Sakaizam-bohitra (...) à Tananarive et à Ambohimanga $»^{21}$, ajoute Julien. Ils sont «investis de pouvoirs très étendus ${ }^{22}$, bien que tous doivent rendre compte directement au Premier ministre. Maires, huissiers et notaires ${ }^{23}$ de leur territoire administratif, les sakaizam-bohitra «perçoivent sur

13 Raison-Jourde (1991).

14 ANOM, 2 Z 27, idem.

15 Raison-Jourde (1991, p. 671).

16 ANOM, 2 Z 27, idem.

17 Raison-Jourde (1991, p. 672).

18 ANOM, 2 Z 27, idem.

19 ANOM, 2 Z 27, idem.

20 Jacob (1994), Rafidison (1986).

21 ANOM, 2 Z 27, idem.

22 Sur les instructions correspondantes à leur charge: H. Raharijoana: «Le centenaire des instructions aux sakaizambohitra 1878-1978 », B.A.M., LVI, 1-2, p. 35-38, cité en note de bas de page par Françoise Raison-Jourde (1991, p. 671).

23 En plus des charges policières, les sakaizam-bohitra sont censés rédiger les Bokimpanjakana ou «Livres du gouvernement», c'est-à-dire la tenue des registres d'état civil (écrits), de la transcription 
tous les actes enregistrés et amendes, des droits fiscaux dont ils gardent une partie ${ }^{24}$. Policiers, ils veillent au maintien de l'ordre, et «signalent les actes d'oppression, d'usurpations et d'exactions de toutes sortes commis par les seigneurs féodaux $\gg^{25}$. La surveillance des populations s'institutionnalise et la dénonciation devient une pratique administrative normale. Parmi les étapes de la construction de l'État en Imerina, cette réforme administrative s'inscrit dans une volonté de réaffirmation de la politique centralisatrice du gouvernement royal. Mais n'ayant aucune instruction et illettrés pour la plupart, les sakaizam-bohitra ne remplissent que rarement leurs obligations. Cette institution est vouée dès ses débuts à l'échec, ce qui fait dire à Julien que «le but recherché fut si peu atteint, qu'en 1881, c'est-à-dire trois ans après, Rainilaiarivony tentait déjà, (...) de remédier à la solution ». Le corps des sakaizam-bohitra sera ainsi «remplacé » trois ans plus tard par les Antily.

\section{Une succession périlleuse}

Le 29 mars 1881, la reine Ranavalomanjaka, Ranavalona II, proclame dans un kabary les «Lois de Madagascar ${ }^{26}$, plus connues sous le nom de «Code des 305 articles ». À mi-chemin entre un code civil et un code pénal malgache, elles renforcent le passage d'un pouvoir oral à un pouvoir qui s'écrit. Éléments tout au moins scripturaux de l'évolution et de la rationalisation de l'État royal et de son administration $^{27}$, elles codifient la vie et inscrivent le pouvoir royal dans l'espace par la création de territoires administratifs qui découpe le royaume en six districts ou provinces $^{28}$. Pour asseoir le contrôle des populations et du territoire et légiférer sur le «gouvernement des hommes», les articles 163 à 173 présentent les fonctions exclusives de police des antily.

Le terme antily se traduit par sentinelle ou surveillant, mais Laisné de la Couronne le traduit en 1881 dans la version française des «Lois de Madagascar» par «agent de police». Françoise Raison-Jourde précise que «la note d'ironie était si générale qu'en 1881, (...), on rebaptisera les sakaizam-bohitra antily, c'est-à-dire sentinelles, éclaireurs, terme qui s'appliquait jusque-là aux colons des confins du royaume occupant les nouvelles terres conquises ${ }^{29}$. L'institution des antily consacre littéralement une «administration» impériale merina à la reconquête du royaume.

Les articles sur les antily sont tous structurés sous la forme crimes-délits/peinesamendes. Ils débutent tous par: «les agents de police pourront arrêter...», ce qui

et de la rédaction des actes et des contrats entre particuliers (ventes d'immeubles, prêts d'argent, donations, testaments, locations de maisons ou de biens-fonds).

24 Raison-Jourde (1991).

25 ANOM, 2 Z 27, idem.

26 ANOM, 2 Z 35, «Lois de Madagascar», traduction manuscrite en français du kabary prononcé par la reine Ranavalomanjaka, Ranavalona II, le 29 mars 1881, faite par Laisné de la Couronne (manuscrit non daté), mais vraisemblablement de l'année 1881. En 1882, est publié l'ouvrage du même auteur, intitulé Les Lois malgaches et les traités, par la typographie La Cloche à Tamatave, Madagascar (sous-série: 2 Z 34).

27 Elles fixent la création d'une administration centrale avec huit ministères, dont les ministères de l'Intérieur et de la Guerre.

28 Ellis (1998).

29 Raison-Jourde (1991). 
permet de détacher une typologie des individus considérés comme devant être réprimés par le pouvoir royal ${ }^{30}$. Véritable code pénal malgache, plus du tiers des articles $\mathrm{du}$ «Code des 305 articles» concerne les questions de police et de justice ${ }^{31}$.

Deux témoins crédules ou ignorants des réalités, qui voyagent à Madagascar dans les années 1880, écrivent: «la police est très bien organisée, à Tananarive et dans les villages principaux, partout enfin où fonctionne une administration régulière; mais son action est à peu près nulle dans tout le reste du royaume, qui échappe à sa surveillance $»^{32}$. Car, en préparation de la première guerre franco-merina (18831885), antily et sakaizam-bohitra se transforment en sergents-recruteurs de l'armée, et «serviront presque uniquement, à partir de 1883, à recruter les conscrits » ${ }^{33}$ explique Raison-Jourde. En 1888-1889, les antily sont «remplacés» par les goverinora madinika, les «petits gouverneurs », futurs chefs de canton des Français ${ }^{34}$. Rainilaiarivony publie à cet effet les règles de ces gouvernements avec de véritables fonctions administratives. Point crucial pour lutter contre les abus, les governora madinika sont «payés» et les fokon'olona ${ }^{35}$ ont un budget. Par ce biais, l'administration royale transfère au fokon'olona les pouvoirs de police des «autorités traditionnelles », notables locaux et tompomenakely.

\section{L'imposition de la responsabilité policière aux communautés villageoises, les conventions de fokon'olona}

Au cours de cette période de rétablissement et de réaffirmation du pouvoir central sur le territoire royal, l'institution sociale du fokon'olona, à l'origine base de l'organisation sociale des Hauts plateaux, devient, sur l'impulsion de ce même pouvoir, une véritable institution politique de régulation de la vie communautaire des populations. Grâce à cette institution sociale, le pouvoir royal de Tananarive maintient une tutelle étroite sur les populations et les communautés villageoises. Car elles

30 ANOM, 2Z35, Sont susceptibles d'être arrêtés «tout individu en état de vagabondage», «tous voleurs de profession», «tous voleurs avec effraction», «tous ceux qui commettraient quelques méfaits», «les chefs de bandes de voleurs ou de malfaiteurs», «les propriétaires des maisons dans lesquels se réunissaient les malfaiteurs » (art. 163); «ceux qui provoqueront du tumulte dans les rues ou les marchés», «ceux qui seraient accusés ou soupçonnés d'être des malfaiteurs, ceux qui seraient porteurs d'objets perdus ou volés, ainsi que ceux qui (...) s'opposeraient à l'exécution de la police / ou / (...) les agents de police de leurs fonctions» (art. 164) et «si une personne fait des démonstrations hostiles au gouvernement, les soldats ont le devoir de l'arrêter» (art. 167).

31 ANOM, 2Z35, «les 12 grands crimes suivis de la peine capitale et la confiscation des biens sans distinction de sexe » (articles 1, 2 et 3), «les Meurtres» (art. 4, 5 et 6), «Les crimes passibles de 10 ans de fer et au-dessus» (art. 8 à 21), «les Vols» (art. 22 à 38), «Lèpre et Variole» (art. 67 et 68), «les Condamnés» (art. 134 à 142), «les Perturbateurs» (art. 143 à 148), «Police» (art. 163 à 173), «les Ministres et les Fonctionnaires» (art. 182 à 188), «Lois relatives aux procès et aux jugements: les Juges» (art. de 189 à 212) et «les Procès » (art. 213 à 265).

32 Le Chartier, Pellerin (1888).

33 Raison-Jourde (1991).

34 À l'époque coloniale, le chef de canton ou gouverneur madinika est le représentant de l' «administration indigène» dans le district, composé chacun de plusieurs cantons (environ 80 districts et plus de 700 cantons à Madagascar). Pour l'administration coloniale française, il est la cellule de base de cette administration.

35 Condominas (1960). Le fokon'olona représente traditionnellement la communauté villageoise liée par la parenté et/ou l'appartenance à un même territoire, le foko, et le droit au tombeau. Le fokon-tany représente la réalité territoriale du foko'nolona. 
détiennent désormais la responsabilité d'assurer la «bonne police» des gens, c'està-dire une forme d'auto-gouvernement des populations dans le respect des règlements édictés. Elles remplissent des tâches administratives de base pour le compte de l'État, assurent la police et l'ordre public, ainsi que l'exécution des tâches de basse justice $^{36}$. Sous l'administration royale et coloniale française, les mpiadidy ${ }^{37}$ sont investis d'une autorité de police sur les populations.

Signe de la modernisation de l'État malgache, en 10 ans, trois institutions de «police» se sont succédées, mais les deux premières, les sakaizam-bohitra et les antily, portent en elles les fruits de leur échec. Néanmoins, leur création ou leur résurgence sont la marque d'une volonté du Premier ministre de réaffirmer son pouvoir sur les territoires de l'Imerina, à une période où l'influence étrangère se fait de plus en plus pressante. Aux formes militaro-policières de domination royale se succèdent de «nouvelles » formes de policing, de «nouveaux » dispositifs militaro-policiers qui reprennent les «traditions» locales du policing royal. La reprise premièrement de l'institution du fokon'olona par le nouvel Ordre, et deuxièmement des modes de régulation des populations au niveau local, est la marque d'une continuité qui transcende les ruptures conjoncturelles d'autorités dominantes. L'institution du fokon'olona devient l'instrument et le relais direct du contrôle de l'administration coloniale française sur les populations des Hauts plateaux.

\section{LES DISPOSITIFS COLONIAUX DE MAINTIEN DE L'ORDRE, UNE «NOUVELLE «POLICE»»"}

\section{Le corps d'occupation et les prémices d'une police coloniale française à Tananarive, octobre 1895-janvier 1896}

Le corps expéditionnaire français débarque à Majunga en mars 1895 avec son artillerie juridique et sa gendarmerie, la prévôté du corps expéditionnaire, régie par le décret portant règlement sur l'organisation et le service de gendarmerie ${ }^{39}$. Datée du 23 mai 1895, faite à Majunga, une note écrite à la plume au bas de l'article 536 de ce décret stipule que «l'arrêté du général en chef en date du $1^{\text {er }}$ décembre 1895

36 ANOM, 2 Z 30, «Conventions de fokon'olona des cantons de Tananarive soumises aux autorités administrative et militaire au sujet: Sécurité publique, insérée dans le «Ny Gazety Malagasy» ${ }^{\circ} 49$ du 8 mai 1885 », en langue malgache. Une traduction du malgache au français de ces conventions permettrait d'expliciter les fonctions de «basse justice» du fokon'olona par des exemples.

37 Pour l'administration le mpiadidy est le représentant des villageois du fokon'olona. Mais pour les populations malgaches, il est le plus bas des fonctionnaires de l'administration coloniale. Souvent illettrés, les mpiadidy sont secondés par les mpikarakara, secrétaires lettrés de la période royale, base de la bureaucratie merina.

38 Académie des Sciences d'Outre-Mer (ASOM), You 212, Notes du résident général Laroche relatives à son administration de janvier à septembre 1896, écrites après la lecture de l'ouvrage d'André You. Elles ont servi en outre à You pour la rédaction définitive de son incontournable ouvrage, Madagascar (Histoire, organisation, colonisation), Berger Levrault, Paris, 1905, 631p.; préfacé par Albert Decrais, ancien ministre des Colonies, et introduit par Gallieni. «Grand colonial», juriste de formation, André You est sous-directeur faisant fonction au ministère des Colonies et professeur à l'École Coloniale. Selon Gallieni, You a mis en lumière «des concepts qui contribueront, à fixer notre doctrine coloniale, de façon à fournir, plus tard, les éléments de solution». You participe de l'acte colonial en tant que théoricien du droit colonial.

39 Décret du $1^{\text {er }}$ mars 1854. 
déclare que les Français et les étrangers résidant à Tananarive sont considérés comme étant à la suite de l'armée et soumis à la juridiction précitée ${ }^{40}$. Toute la population française et étrangère ${ }^{41}$ de la capitale royale nouvellement conquise, le 30 septembre 1895, est soumise aux mêmes régulations que les «vivandiers, les cantiniers, industriels et leurs domestiques, autorisés à suivre l'armée ${ }^{42}$. Une première forme de juridiction militaire d'exception s'applique aux Français civils et aux ressortissants étrangers. Elle impose en actes la nouvelle autorité conquérante.

La gendarmerie, sous la forme d'un corps prévôtal expéditionnaire, devient la prévôté du corps d'occupation, organisée à Tananarive par le décret du 9 octobre 1895. Le prévôt du corps, le chef d'escadron Gaudelette, débute alors un intense travail préparatoire sur deux projets, l'un relatif à la «Constitution et fonctionnement de la Police à Tananarivo » ${ }^{43}$ et l'autre à l'organisation de ce service ${ }^{44}$. Fruit de trois semaines d'observations et de pratiques, ce projet a subi, selon lui, «l'épreuve de l'expérience et a donné de bons résultats ${ }^{45}$. Deux annexes, datées du 20 octobre, le complètent l'une sur la «Constitution de la Police à Tananarivo», et l'autre sur l' «Organisation de la Police à Tananarivo $»^{46}$. Le texte fondamental du 8 octobre se divise en onze $« \operatorname{articles}{ }^{47}$. Il propose de diviser la ville de Tananarive en $12 \mathrm{sec}$ teurs, «chacun de ces secteurs comprend un ou plusieurs quartiers et à chaque secteur correspond un poste de police occupé par 20 agents indigènes commandés par deux officiers indigènes ${ }^{48}$. Ces projets maintiennent donc «la police malgache» qui «relève directement de son chef (...) responsable de la manière dont le service est effectué ${ }^{49}$.

Tout comme l'avènement de la mainmise française sur les Hauts plateaux maintient la monarchie merina et l'organisation administrative malgache à Tananarive et ses environs à l'automne 1895; un double dispositif de police malgache et français s'impose tout en reprenant l'institution antérieure des mpiadidy. La police malgache est «spécialement chargée d'assurer, de jour comme de nuit, la tranquillité de la ville, la sécurité des habitants, d'arrêter les délinquants et les criminels malgaches,

40 ANOM, 6(2)D4, Document imprimé à Majunga et daté du 23 mai 1895: «Art. 536 du décret du $1^{\text {er }}$ mars 1854, Corps expéditionnaire de Madagascar, «Règlement de Police concernant les vivandiers, les cantiniers, industriels et leurs domestiques, autorisés à suivre l'armée»».

${ }^{41}$ Ellis (1998, p. 60). Vers 1894, «on pouvait dénombrer environ 3300 résidents européens à Madagascar, dont 2850 vivaient sur la côte est».

42 ANOM, 6(2)D4, idem.

43 ANOM, 6(2)D4, Projet du chef d'escadron Gaudelette: «Constitution et fonctionnement de la Police à Tananarivo » daté du 8 octobre 1895 .

44 ANOM, 6(2)D4, Lettre du chef d'escadron Gaudelette, prévôt du corps expéditionnaire à M. le général chef d'état-major, Police de Tananarive, le 21 octobre 1895.

45 ANOM, 6(2)D4, idem.

46 ANOM, 6(2)D4, idem.

47 ANOM, 6(2)D4, «la Police malgache», «la Police française» dont font partie les «Inspecteurs ordinaires» (c'est-à-dire les «Inspecteurs indigènes») au nombre de douze, salariés par les autorités françaises, «tenue», «Inspecteurs principaux et auxiliaires », un «interprète» «attaché spécialement au chef d'escadron prévôt», «salaires», «mpiadidy» (chef de quartier), «gouverneurs des villages et mpiadidy de la banlieue», «chef de la police malgache», «prisons» et les «consignes pour les prisons».

48 ANOM, 6(2)D4, Projet du chef d'escadron Gaudelette: «Constitution et fonctionnement de la Police à Tananarivo » daté du 8 octobre 1895 .

49 ANOM, 6(2)D4, idem. 
de veiller à la propreté des rues, des abords des maisons et des marchés, de faire exécuter les prescriptions des autorités malgaches approuvées par l'autorité française, de signaler sans retard aux agents de cette autorité les délits et crimes commis par les personnes qui ne seraient pas des sujets malgaches, de prêter son concours à la Gendarmerie en toute circonstance, de donner l'alarme et de participer aux secours en cas d'incendie ${ }^{50}$. Tranquillité, sécurité et salubrité sont les maîtres mots de cet enchevêtrement policier.

Ce double dispositif franco-malgache se retrouve à chaque niveau de la hiérarchie policière. En effet, au plus haut niveau bureaucratique hova, l'ancien ministre de l'Intérieur, Rainitsimbazafy, remplace le 14 octobre 1895 le Premier ministre Rainilaiarivony, écarté du pouvoir et placé en résidence surveillée le 2 octobre. Un nouveau gouvernement malgache est constitué dans lequel Rainandriamanpandry ${ }^{51}$ (ancien gouverneur hova de Tamatave) prend le portefeuille de l'Intérieur, jusqu'à son procès et son exécution pour «trahison» en octobre $1896^{52}$. Dans la continuité de l'administration de la sécurité royale et des dispositifs de police vernaculaires mais passés sous domination française, ce dispositif dual correspond au moment du «protectorat».

La «prévôté» du chef d'escadron Gaudelette constitue en partie les cadres de la «nouvelle police ${ }^{53}$ voulue par le résident général Hippolyte Laroche. Le 29 décembre 1895, trois semaines avant ce dernier, l'arrivée de policiers professionnels venant de la métropole est signalée dans les archives, sans précision sur leur service d'origine ${ }^{54}$. Cinq fonctionnaires de la police spéciale de la résidence générale débarquent dans la colonie. Un commissaire spécial ${ }^{55}$, un chef de service, un commissaire-adjoint et deux inspecteurs composent cette équipe de policiers métropolitains. L'un de ces derniers reste à Tamatave pour y remplir les fonctions de commissaire de police, les autres sont affectés à Tananarive où ils procèdent à l'organisation du service, tant municipal que de sûreté publique, et prennent également la charge des prisons civiles. Au-delà de la prévôté, ces fonctionnaires de police constituent les cadres de la future sûreté générale.

\section{La "protexation» ${ }^{56}$ et la police "civile» du résident général, janvier 1896-septembre 1896}

Dès son arrivée à Madagascar le 18 janvier 1896, le résident général Laroche affirme - dans ses notes - sa préoccupation pour «la création de milices ${ }^{57}$ placées

50 ANOM, 6(2)D4, idem.

51 Esoavelomandroso (1979).

52 Ellis (1990). L'introduction de cet ouvrage s'ouvre sur cette phrase: «Cette histoire se lit comme un roman policier, mais les personnages sont réels ».

53 ASOM, (Académie des Sciences d'Outre-Mer), You 212, Notes du résident général Laroche.

54 ANOM, 5(1)D1, Rapport annuel pour l'année 1904 sur la police administrative et judiciaire et le service pénitentiaire (rapport remis en février 1905).

55 Bulletin du Comité de Madagascar, $2^{\text {ème }}$ année - n 1 - janvier 1896, p.18.

56 Paillard (1994). D’après, Moor, Wesseling (1989, p. 187), la «protexation» est un mot forgé par un journaliste londonien à partir des deux mots «protectorat» et «annexion».

57 ASOM, You 212, idem. 
directement sous ses ordres. Aidé par le commissaire spécial, fraîchement arrivé, Laroche «poursuit» l'œuvre du chef d'escadron Gaudelette. Il mobilise d'anciens soldats malgaches de l'armée royale merina appartenant à la «police du palais», la garde prétorienne, dévouée et certainement toujours fidèle, de la reine. Ces soldats sont encadrés par une trentaine de caporaux et de soldats français du corps d'occupation. Mais, «pour les armer, le général Voyron ne put délivrer que des mousquetons sans baïonnettes aux quelques 300 miliciens de la nouvelle «police» ${ }^{58}$. Sur l'île-continent, cette «force» ne peut donc jouer qu'un rôle «effacé » ${ }^{59}$, note Laroche. Cette lucide constatation paraît justifier a posteriori son incapacité à structurer l'embryon de police initié par Gaudelette à Tananarive et à juguler l'insurrection naissante ${ }^{60}$. Cette milice joue tout de même, insiste Laroche, « un rôle significatif ${ }^{61}$.

Fruit de l'antagonisme entre pouvoirs civil et militaire en matière d'ordre, cette milice représente la garde rapprochée de Laroche, à son unique service, sans structuration réelle et cohérente d'un dispositif de police. «Elle rendit de précieux services par sa mobilité, assurant à elle seule la protection des courriers et d'un certain nombre de villages $»^{62}$, constate-t-il, sans plus de précision.

Néanmoins, une volonté d'institutionnalisation des dispositifs de polices, à défaut de structuration, apparaît sous la résidence générale Laroche. Le décret du 11 juillet 1896, puis un arrêté local du 6 novembre 1896, organise la milice indigène sous le nom de «Garde Indigène». Ce décret met fin théoriquement à une seconde dualité des pouvoirs en matière de maintien de l'ordre, pouvoirs militaire avec la prévôté et civil avec le résident général et le commissaire spécial. Il rend «applicable à Madagascar la répartition d'attributions instituée en Indochine par les décrets des 27 janvier 1886 et 3 février 1890, et la désignation d'une autorité unique cumulant les attributions civiles et militaires ${ }^{63}$. Un feuillet griffonné au crayon de bois, non daté mais présent dans les papiers du chef d'escadron Gaudelette, mentionne «voir organisation police Indo-Chine et autres colonies » ${ }^{64}$. Dans le cas de Madagascar, la recherche de précédents administratifs et juridiques pointe très souvent la colonie d'Indochine comme modèle impérial ${ }^{65}$. Au printemps et à l'été 1905 , une intense activité juridique occupe les bureaux du gouvernement général à propos de la création d'un bureau militaire à Madagascar. Le commandant Leblond, chef du bureau militaire à Saïgon, fournit aux autorités de Madagascar les renseignements sur le fonctionnement du bureau militaire du gouvernement général de l'Indochine ${ }^{66}$. De multiples formes de circulations de personnels, de techniques, de savoirs administratifs et organisationnels peuvent être énoncées d'une part entre la

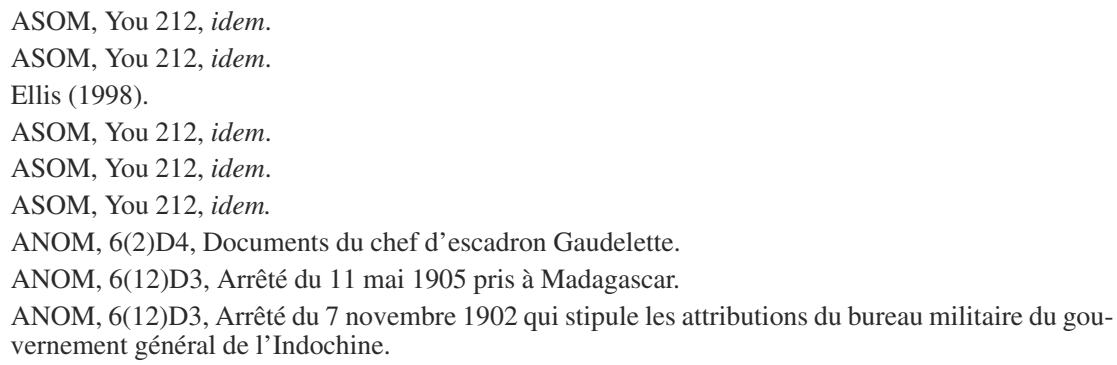


métropole et l'empire et d'autre part à l'intérieur des frontières impériales ${ }^{67}$. Ces circulations structurent, tout comme les réalités malgaches, les dispositifs de polices à Madagascar, bien que par la suite une adaptation aux réalités et aux particularités du territoire colonisé nuance ce constat.

Ainsi, les faibles acquis de la conquête sont compromis par l'insurrection des Menalamba $^{68}$. Quelle solution envisage Paris? En finir avec le «fantôme d'un protectorat ${ }^{69}$ et user de la manière forte. Le parti de l'ordre fait appel à un colonel d'infanterie de marine Joseph Gallieni ${ }^{70}$, «qui s'est déjà fait remarquer par de brillantes actions au Sénégal, au Soudan français et en Indochine ${ }^{71}$. Gallieni est promu général de brigade puis général de division pour l'occasion et investi dans la soirée du 28 septembre 1896 des doubles fonctions de gouverneur général et de commandement supérieur des troupes ${ }^{72}$. Les pouvoirs civil et militaire sont désormais entre les mains d'un seul homme; le général Gallieni a été pourvu «d'une autorité absolue sur tous les services de l' $̂$ lle sans exception ${ }^{73}$.

\section{De la répression totale à un "État complet» ${ }^{74}$, "Gallieni Pacificateur»"}

Portée à son stade suprême sous le proconsulat du premier goverinora masiaka, «gouverneur méchant», de Madagascar le général Gallieni, la Maxime de l'ordre est l'enseigne de la situation coloniale ${ }^{76}$. "C'est par la fermeté de l'énergie que l'ordre va être établi», ajoute Laroche ${ }^{77}$. Fermeté de l'Ordre nouvellement souverain légitimée par le fait que «l'insurrection était à peu près générale ${ }^{78}$.

Arrivé le 16 septembre à Tananarive, Gallieni incarne la victoire de la stratégie de l'ordre. L'usage de la force apparaît crucial pour la sauvegarde de l'Ordre colonial balbutiant. Avec son triptyque coercition-répression-persuasion, Gallieni applique à Madagascar des théories et des méthodes rodées auparavant dans d'autres territoires impériaux, qui se diffuseront grâce à Lyautey en Algérie et au Maroc. Corollaire de l'Ordre colonial, la volonté d'un «État complet» émerge dès le début de son plan de répression de l'insurrection, la «pacification», selon l'euphémisme habituel. Pour Gallieni, l'affirmation d'un État colonial viable ne s'envisage pas sans le rétablissement de l'ordre par tous les moyens. La répression totale de l'insurrection des Menalamba ${ }^{79}$ et la soumission des chefs rebelles au nouvel

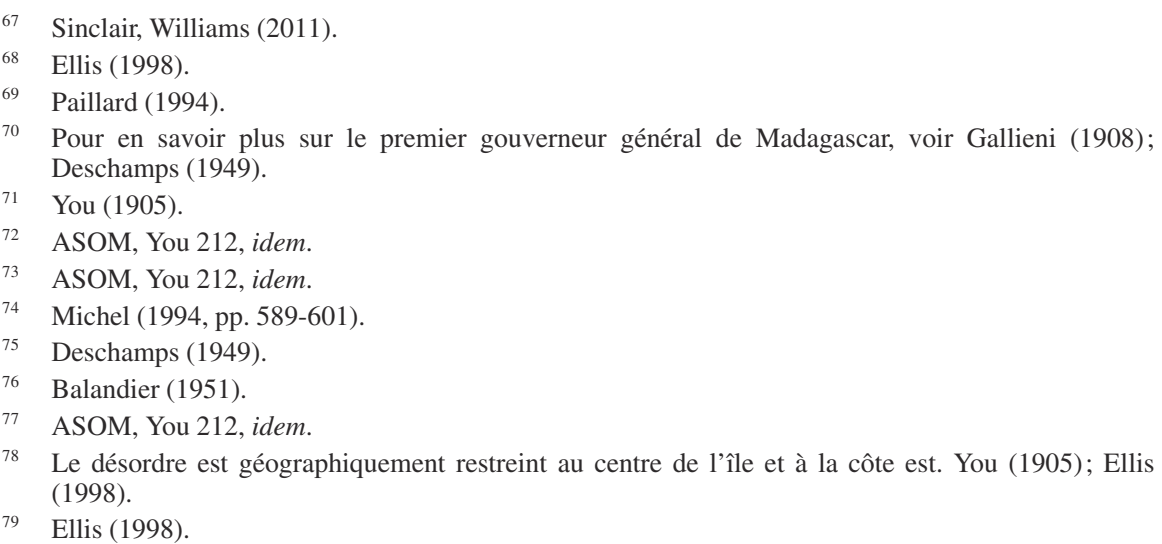


Ordre ne peut se lire qu'à travers ce schéma d'imposition de la domination coloniale ${ }^{80}$ française, préalable à l'assujettissement des populations. Or, comment les dispositifs de polices naissants s'intègrent-ils dans ce processus d'imposition par la force de la domination française?

Des «Mesures d'ordre militaire et de Haute Police ${ }^{81}$ sont promulguées par les arrêtés du résident général. Ce dernier se fonde sur l'annexion de Madagascar et déclare l'état de siège dans les régions centrales. Tout d'abord, des «mesures d'exécution d'ordre administratif et militaire» consacrent l'organisation en territoires et en cercles militaires de tout le nord de l'Imerina, avec constitution pour Tananarive et sa proche périphérie d'un «gouvernement militaire spécial», et la proclamation de l'état de siège dans l'Imerina et le Betsiléo. La loi martiale est déclarée, tout acte suspect est de ce fait passible du tribunal militaire. Enfin, l'application du système de la «Tache d'huile ${ }^{82}$ impulse une tactique militaire coloniale de reconquête progressive du territoire.

Dans le même temps, Gallieni augmente les effectifs des «forces de la police indigène» qui est provisoirement replacée «sous la direction du commandant de la prévôté; elle ne reprit son autonomie que quelques mois plus tard ${ }^{83}$. La Garde indigène supplée ainsi au manque endémique d'effectifs européens de l'armée d'occupation. Au-delà de cette nécessité primaire, l'usage de la Garde indigène par Gallieni s'explique, selon l'historienne Chantal Valensky, essentiellement pour des raisons financières et budgétaires. Pour Gallieni, «la part à donner aux miliciens et aux tirailleurs devient une option cruciale (...), il semble assuré que Gallieni accorde l'essentiel de ses soins à la milice, pour la raison qu'elle représente pour lui sa véritable force de manœuvre car payée sur le budget local. Il en use à discrétion alors que pour l'usage des tirailleurs, il est redevable au ministère de la Guerre ou au Parlement ${ }^{84}$, note Valensky. En effet, la milice dite «supplétive» n'est pas soumise au contrôle du Parlement sinon indirectement.

Au cours de ce moment de reconquête, la Garde indigène est indéniablement l'instrument du maintien de l'ordre colonial dans les territoires nouvellement soumis. L'arrêté local du 6 novembre 1896, déjà cité, stipule le rôle d'escorte (protection des convois et des courriers), de protection des chantiers des travaux publics, des marchés, des dépôts d'approvisionnement des gardes indigènes et l'action commune entre la milice et les villages armés considérés comme «fidèles», mais passe sous silence leur totale participation aux opérations militaires de reconquête. Les opérations de «pacification» sont qualifiées d' «opérations de police» et très rarement d' «opérations de guerre». La conquête étant en théorie achevée, la charge des opérations incombe en grande partie au budget local, et donc à la Garde indigène et à leurs indispensables «supplétifs», les partisans. Ce sont des «opérations de

\footnotetext{
80 Harmand (1919 [1910]).
}

81 Arrêtés du 27 septembre 1896.

82 Du centre vers la périphérie, les «insurgés» sont repoussés de manière centrifuge. Dans les territoires «pacifiés» des «postes», de plus en plus avancés, sont construits afin de quadriller le nouvel espace occupé au fur et à mesure de l'avancée des troupes. La politique de la «Tache d'huile » diffuse progressivement l'état de siège à d'autres régions de l'île, Tamatave en 1897, dans le pays sakalave et à Farafangana en 1898.

83 You (1905, pp. 401-402). Les premières données statistiques fiables sur les effectifs de la Garde indigène datent de 1907.

84 Valensky (1995, p. 137). 
police», par opposition aux «opérations de guerre» de la conquête, même si les militaires (infanterie de marine, légion étrangère, tirailleurs sénégalais et malgaches) participent aux opérations et bien que les pratiques soient davantage perçues comme «guerrières» et non «policières». Le maintien de l'ordre guerrier ou le rétablissement de l'ordre par la force des armes symbolisent l'avènement de la pax gallica.

\section{Le corps des partisans, une police informelle}

Page d'ombre de l'historiographie coloniale, les agents des forces dites «supplétives », le corps des partisans, jouent néanmoins dans les faits un rôle incontournable. À travers la décision $198^{85}$ du 13 avril 1897, signée par Gallieni, le pouvoir colonial crée un corps de partisans de l'Imerina. Suit l'arrêté du 19 février 1898 qui autorise les commandants de cercle et les chefs de province à organiser des corps de partisans dans leur circonscription. Le budget des partisans est réglé sur le budget des prestations (travail forcé), une journée de service comme partisan équivaut à deux jours de prestations pour l'administration civile. Armés de fusils ou de carabines modèle 1874, portant une coiffe reconnaissable, «une bande d'étoffe rouge fixée au chapeau » ${ }^{86}$, le corps des partisans est complémentaire des «villages armés » dont la fidélité était certaine. C'est le principe de la «société en armes», cher aux doctrines clausewitziennes, déjà utilisé à l'époque royale avec la militarisation de la société grâce à l'institution des «chefs de cent et des chefs de mille» ${ }^{87}$.

Parlant de Gallieni, You insiste sur le fait que «la plus grande part de son activité fut la répression de l'insurrection ${ }^{88}$. C'est au cours de la pacification et de l'imposition de la pax gallica, que se font jour les réalités de cette «nouvelle police française» et l'indistinction entre les dispositifs militaire et policier de défense intérieure de la colonie de Madagascar. Un processus d'institutionnalisation des dispositifs de polices au sein de l'État colonial et de manière synchronique une volonté de détachement de la hiérarchie militaire commence à la fin de la période Gallieni. L'intense activité juridique de l'année 1903 en ce qui concerne la Garde indigène annonçait le décret du 13 janvier 1904 supprimant la gendarmerie à Madagascar et le $15^{\text {ème }}$ régiment d'infanterie coloniale.

85 ANOM, 6(2)D11, Dossier sur le corps des partisans 1897-1906. Un travail spécifique est en cours.

86 ANOM, 6(2)D11, Article 5 de la décision 198 du 13 avril 1897.

87 Raison-Jourde (1991, pp. 383 et 418). «Radama Ir avait en effet quadrillé les ruraux en vue de la corvée avec des chefs de cent et des chefs de mille, qui étaient autant d'autorités individualisées ». Le corps des partisans institué par Gallieni reprend de nouveau la tradition monarchique du contrôle rural des populations et se fonde sur cette ancienne institution. Pour mieux comprendre les pratiques de contrôle et de régulation des populations au plus près du terrain par cette police, l'étude du corps des partisans et leurs rapports avec la Garde indigène pourraient apporter de nombreux éclairages.

88 You (1905, p. 404). 


\section{L'INSTITUTIONNALISATION LENTE DES DISPOSITIFS DE POLICES A MADAGASCAR}

\section{Une triple modernité policière et coloniale?}

Bien que l'illusion d'une «modernité» perceptible dans l'instauration des polices à Madagascar soit enfermée dans les cadres d'une domination coloniale, l'émergence d'une police urbaine, administrative et judiciaire, dans le sens métropolitain du terme, peut être considérée comme une modernité urbaine à Madagascar. Elle n'est organisée que «dans les grands centres, érigés en communes ou en centres autonomes ${ }^{89}$. En 1905, cinq centres urbains, Tananarive, Diego-Suarez, Tamatave, Majunga et Nosy-Be, existent à Madagascar. Ils sont neuf en $1912^{90}$, avec en sus Fianarantsoa, Sainte-Marie, Andovoranto, Mananjary ${ }^{91}$. Lieux privilégiés de la présence militaire, policière et administrative française, ces espaces reflètent l'existence visible, sûre et assurée de l'Ordre colonial régnant. La police est quant à elle entièrement assurée hors de ces centres par des agents de la Garde indigène exerçant les fonctions de commissaire de police. Mais cela ne signifie pas a contrario que la Garde indigène soit absente de ces grands centres.

You désigne par les termes de «police administrative et judiciaire ${ }^{92}$ les forces de police mises en place dans ces centres urbains et en premier lieu Tananarive, le centre névralgique du pouvoir colonial. En 1898, à la fin de l'état de siège à Tananarive, le commissaire spécial chargé des questions de sûreté intérieure est nommé commissaire central et chef du service pénitentiaire de Tananarive. Deux arrêtés locaux, du $1^{\text {er }}$ novembre 1901 et du 9 juillet 1903, régissent les questions concernant «le personnel européen et indigène ${ }^{93}$ de cette police urbaine. Elle comprend un chef du service de sûreté, des commissaires centraux, des commissaires, des inspecteurs principaux, des inspecteurs, des brigadiers et des agents ${ }^{94}$. Les commissaires de police de Madagascar sont nommés par décisions du gouverneur général suivant les instructions générales du 24 décembre $1902^{95}$. Le personnel indigène de la police administrative et judiciaire est organisé quant à lui par un arrêté local particulier du 28 novembre $1900^{96}$. Ce personnel forme la majorité des effectifs subalternes du dispositif policier qui, d'après You, représente environ $76 \%$ des effectifs en 1905. Il compose les deux dernières catégories de personnel, brigadiers et agents ${ }^{97}$. Signe de l'essence militaire du dispositif de polices à Madagascar, les agents indigènes sont

\footnotetext{
89 You (1905, p. 402).

90 ANOM, 5(1) D 2, État nominatif des circonscriptions où fonctionne un service anthropométrique, 1912.

91 ANOM, ibid.

92 You (1905, p. 401).

93 You (1905, p. 401).

94 You (1905, p. 402). Les articles 5 et 11 de l'arrêté du 11 novembre 1901, partiellement modifiés par l'arrêté du 9 juillet 1903, déterminent les conditions de recrutement et d'avancement du personnel européen de la police.

95 You (1905, p. 403).

96 You (1905, p. 402).

97 Il faut attendre les années 1930 et 1940 pour voir accéder aux postes d'inspecteurs et d'inspecteurs principaux de police le personnel indigène.
} 
recrutés «uniquement parmi les anciens militaires, conducteurs auxiliaires de l'artillerie, tirailleurs malgaches ou gardes régionaux; la préférence est réservée aux gradés $\gg^{98}$.

Il est possible de dire que la police administrative et judiciaire urbaine à Madagascar est en partie le fruit d'apports exogènes sous la forme d'une transposition dans les colonies des dispositifs policiers existant en métropole. You affirme dans ce sens que les attributions des commissaires de police sont en tout point identiques à celles de leurs homologues métropolitains ${ }^{99}$. L'expression d'un État jacobin colonial s'exprime dans la constitution des dispositifs de polices dans la grande Île, où «le fonctionnement général de la police municipale de Tananarive, (...), s'applique également aux autres villes de la colonie qui ont une organisation analogue, en tenant compte de l'importance de la population et de sa composition ${ }^{100}$.

\section{Une Sûreté centralisée?}

En 1903, la Sûreté, le pouvoir central policier, n'a de centralisé que le bureau du Chef de la sûreté à Tananarive qui relève directement des bureaux du gouverneur général. Il est intégré au bureau militaire du gouvernement général dès sa création en $1905^{101}$. Ce dernier détient les prérogatives budgétaires de la Garde indigène et définit le caractère des opérations de "police». Sous Gallieni, le commissaire général de Tananarive exerce les fonctions de chef de la sûreté et de chef du service pénitencier de la région centrale.

Le chef du service de la sûreté est en théorie le chef de l'ensemble des dispositifs policiers de sécurité intérieure et d'enfermement carcéral de la colonie. Il a sous son autorité la police administrative et judiciaire et la Garde indigène, la police spéciale des chemins de fer ${ }^{102}$, la police de sûreté, l'anthropométrie et l'identification, les maisons d'arrêt et de justice, de force et de correction. Il est censé être chargé de la centralisation des renseignements de sûreté; mais elle ne sera effective que bien plus tard. Élément nuançant la réalité jacobine de l'État colonial naissant, ce n'est qu'en 1912, qu'une véritable centralisation des renseignements sera demandée, en effet «les divers commissaires de police de la colonie qui jusque-là n'avaient pas été tenus à signaler les événements survenus dans leurs circonscriptions à leurs collègues, en service dans les provinces voisines, sont astreints actuellement à donner à ces derniers communication des faits de cette nature qu'ils doivent signaler également à la sûreté générale ${ }^{103}$. Cette mesure a été étendue aux agents de la Garde

98 ANOM, 5(1)D1, Rapport annuel de la Sûreté pour l'année 1904: «Police administrative et judiciaire et service pénitentiaire», février 1905.

99 You (1905, p. 403).

100 ANOM, 5(1)D1.

101 ANOM, 6(12)D3, Dossier sur la création du bureau militaire du gouvernement général de Madagascar, mai-décembre 1905.

102 Berlière (1996). En France, la «police spéciale des chemins de fer» se consacre essentiellement au renseignement administratif et politique. À Madagascar, la construction de la ligne Tananarive-Côte Est (TCE), longue de 366 km, commence à Brickaville en 1900 et atteint Tananarive en 1909. La liaison Brickaville-Tamatave est achevée juste après. Voir Fremigacci (2006).

103 ANOM, 5(1)D2, Rapport annuel de la Sûreté générale pour l'année 1912 (rapport remis le 13 mai 1913). Le gouverneur général Albert Picquié (1910-1914) passait, aux yeux des colons, pour un maniaque du renseignement et de la surveillance policière (précision apportée par Jean Fremigacci). 
indigène ${ }^{104}$. Le chef de la sûreté doit enfin veiller à la tenue du casier central de police ${ }^{105}$, et gérer le «service d'identité judiciaire», et diriger les services anthropométriques.

\section{La modernité anthropométrique coloniale}

La véritable illusion de modernité des dispositifs de polices à Madagascar, poudre aux yeux des chefs successifs de la sûreté, convaincus de faire œuvre de précurseurs aux colonies tout au moins, est le service d'anthropométrie judiciaire. Ce service est reconnu comme une branche officielle de l'administration pénitentiaire, comme à son origine en métropole. À longueur de rapports, les chefs de la sûreté dissertent sur ces nouvelles technologies policières pour la colonie. Jusqu'à la veille de la Première Guerre mondiale, les rapports annuels de la Sûreté générale sont extrêmement minces: hormis la capitale, elle n'avait que peu de renseignements sur le reste de la colonie et les questions de police étaient décentralisées dans les provinces civiles et les territoires militaires. Ces rapports sont en revanche prolixes au sujet du service anthropométrique.

Le service anthropométrique et d'identification, créé à Tananarive d'après les énoncés scientifiques et les méthodes d'Alphonse Bertillon ${ }^{106}$, fonctionne à partir du $1^{\text {er }}$ décembre 1902, mais faute de personnels initiés aux procédures, «ce n'est que vers le mois de mai 1903 (...) qu'il y a eu suffisamment d'anthropomètres instruits aptes à accomplir un travail efficace ${ }^{107}$. Dix-sept ans après le décret du 6 mars 1895 portant création, en métropole et pour les policiers parisiens, du «cours dit de signalement et de reconnaissance anthropométriques à l'usage des agents relevant du service de la Sûreté, du service des garnis, des brigades de Recherches et du Contrôle Général » ${ }^{108}$, un cours permanent d'anthropométrie judiciaire est créé à Tananarive par l'arrêté du 5 février $1912^{109}$, avec la formation d'élèves «mensurateurs-identificateurs ». Les formalités anthropométriques avec photographie judiciaire sont pratiquées uniquement à Tananarive et Diego-Suarez en $1912^{110}$. En 1916, toutes les provinces de la colonie possèdent un poste anthropométrique situé généralement au chef-lieu de la circonscription: Diego-Suarez, Majunga et Tamatave; Fianarantsoa, Mananjary, Nosy-bé, Sainte-Marie (police administrative). Les autres postes sont sous la direction des agents de la Garde indigène ${ }^{111}$. En 1916, «90 000 fiches tant anthropométriques qu'alphabétiques ${ }^{112}$ sont recensées au casier central de Tananarive, alors qu'en 1905 le service anthropométrique de la rue Scipion d'Alger

\footnotetext{
104 ANOM, 5(1)D2, idem.

105 You (1905, p. 403).

106 Cf. le «projet Bertillon» sur [http://www.criminocorpus.cnrs.fr/article639.html].

107 ANOM, 5(1)D1, Rapport annuel de la Sûreté pour l'année 1904: «Police administrative et judiciaire et service pénitentiaire», rapport remis en février 1905.

108 Rapport de la Direction générale des recherches (1903, p. 124) cité par López (2006).

109 ANOM, 5(1)D1, idem.

110 ANOM, 5(1)D2, Rapport annuel de la Sûreté générale pour l'année 1912 (rapport remis le 13 mai 1913).

111 ANOM, 5(1)D5, Rapport sur le fonctionnement des divers services intéressant la Sûreté publique de la colonie pendant l'année 1916.

112 ANOM, 5(1)D5, idem.
} 
rassemble déjà cent quarante mille fiches de prévenus et qu'en 1893 à Paris «les sommiers judiciaires, rattachés jusqu'alors à la police judiciaire, comptent près de huit millions de fiches ${ }^{113}$. Au début du $\mathrm{XX}^{\mathrm{e}}$ siècle à Madagascar, le service anthropométrique de la sûreté générale est un dispositif en devenir, essentiellement urbain, qui n'incarne pas les pratiques de police et de maintien de l'ordre en brousse.

\section{Le chef de district, premier policier de la brousse}

Sur le terrain, les dispositifs de police sont largement décentralisés dans les provinces civiles, dirigées par des chefs de province, et les territoires militaires, sous l'autorité du commandant de cercle. Dans leurs rapports trimestriels, les chefs de district commencent toujours par le chapitre intitulé «État d'esprit (des populations), sécurité et ordre public», dont la teneur est généralement lénifiante. De plus, l'arrêté du 13 juillet 1909 place la «police administrative et judiciaire » pour emploi sous les ordres des administrateurs chefs de province ce qui renforce cette délégation très large des pouvoirs voulue par Gallieni. Dans les provinces civiles, le chef de province, et par délégation les chefs de districts, sont donc les premiers policiers de la brousse ${ }^{114}$.

Le chef de district contrôle «la population par la tutelle qu'il exerce sur les fokon'olona, grâce au régime de l'indigénat ${ }^{115}$. Le décret du 9 mars 1902, qui réorganise le fokon'olona, comporte des articles spécifiques sur la police ${ }^{116}$. L'article 25 du décret reconnaît la «responsabilité collective» du fokon'olona devant l'administration. Ainsi, le chef de district contrôle et administre des «collectivités serviles » ${ }^{117}$ sur une base territoriale et non des sujets-individus. Quant au Code de l'Indigénat, il est la base de la justice pénale administrative, confondant judiciaire et exécutif, imposé à Madagascar en 1901 et remanié en 1904, 1908 et 1912. Dans ce dernier, l'arrêté du 4 décembre 1912, l'article 15 rassemble l'ensemble des «infractions passibles des peines disciplinaires ». Cette « répression par voie disciplinaire ${ }^{118}$ caractérise les aspects répressifs du pouvoir judiciaire entre les mains de l'exécutif. Symbole d'une justice coloniale expéditive, le chef de district peut, lui et lui seul, condamner, sans jugement, au titre de l'Indigénat tout «indigène » réfractaire à l'impôt ou ayant commis une infraction au Code.

Au-delà des charges de justice ${ }^{119}$, le chef de district détient des attributions militaires et surtout policières. Il commande la Garde Indigène stationnée dans sa

113 About (2004).

114 Décret du 8 juillet 1906.

115 Fremigacci (1978). Deux procédures disciplinaires spécifiques à Madagascar sont placées entre les mains du chef de district: le décret sur le Fokon'olona du 9 mars 1902 et le Code de l'Indigénat (au Sénégal 1887, imposé à Madagascar en 1901, et remanié en 1904, 1908, 1912, 1924 et 1937). Ce paragraphe se fonde sur le travail de Jean Fremigacci.

116 Article 10: «en matière de police générale», article 11: «en matière de police rurale», et article 12 : «en matière de justice civile».

117 Fremigacci (1978).

118 Ibidem.

119 Cette réglementation est indispensable afin «d'assurer la sécurité par une prompte répression», You (1905, p. 232). You présente ces réglementations juridiques comme des expédients provisoires, mais du provisoire qui dure, et légitime une triple répression disciplinaire par voie administrative, judiciaire et policière entre les mains d'un seul homme. 
circonscription. Appareil de discipline et d'assujettissement au main du pouvoir, les gardes indigènes, bras armé de l'administration civile, en sont les agents d'exécution. Ils font appliquer le Code de l'indigénat.

Commandant la Garde Indigène, le chef de district est également censé en être l'instructeur. Or, «le paradoxe du chef de district» ${ }^{120}$ est qu'il est vu comme un simple «agent d'exécution aux ordres du chef de province», alors que «la situation coloniale fait de lui, en réalité, le rouage essentiel du pouvoir ${ }^{121}$. Quelquefois seul fonctionnaire français présent dans sa juridiction, le chef de district est le plus souvent un jeune «administrateur débutant, de moins de trente ans, et parfois un modeste fonctionnaire du cadre des services civils ${ }^{122}$. Au début de l'année 1911, un chef de district de la province de Mananjary demande aux autorités de lui adjoindre un fonctionnaire européen afin de satisfaire à ses tâches de police ${ }^{123}$. Cette mesure, selon lui, permettrait de le décharger de ses obligations en ce qui concerne la prison, le détachement de la Garde Indigène, certaines tournées de police et la surveillance des travaux. Le cas extrême de ce chef de district illustre parfaitement cet état de fait, il n'a «aucune compétence pour la formation et l'instruction de la Garde indigène » ${ }^{124}$. Ce rapport $\mathrm{a}$, entre autre, permis une prise de conscience des autorités centrales de la colonie, quant à la nécessité de revoir l'exécution du service de police incombant à la Garde indigène, ainsi que les attributions de police dévolues au chef de district. Ce rapport correspond à l'envoi d'une mission d'inspection dans la portion centrale de la colonie, la première semaine du mois de février 1911, et à la transmission de la circulaire ${ }^{\circ} 348$ à tous les chefs de province. L'inspection confirme les dires du chef de district et la circulaire constate qu' «il était à présumer que le service de la Garde indigène à Madagascar n'est pas, en général, assuré comme il devrait l'être ${ }^{125}$. Malgré le décret du 8 juillet 1906, les directives du gouvernement général se trouvent être entravées dans leurs applications sur le terrain. Incarnation de l'Ordre colonial et du commandement policier dans les provinces civiles pour les populations, le chef de district est la «cheville ouvrière» de l'État colonial, de l'administration territoriale civile, et le premier acteur du policing colonial.

\section{CONCLUSION}

Issues de l'institution militaire, appareil d'État, de répression, de régulation et d'ordre, les polices constituent un élément riche en enseignements et un cadre d'élaboration primordial de l'État royal malgache et de l'État colonial français à Madagascar. Ainsi, l'analyse de la construction lente des dispositifs de polices à Madagascar met en lumière premièrement la volonté du gouvernement royal et du

120 Fremigacci (1978).

121 Ibidem.

122 Ibidem.

123 ANOM, 6(12)D5, Rapport politique et administratif sur l'année 1910, fait dans la province de Mananjary, le $1^{\text {er }}$ février 1911.

124 Ibidem.

125 ANOM, 6(12)D5, Circulaire n³48, Gouvernement de Madagascar à M.M. les administrateurs chef de province au sujet de l'application du service de police incombant à la Garde indigène, datée du 8 février 1911. 
Premier ministre Rainilaiarivony d'asseoir de nouvelles formes d'Ordre dédiées entièrement à sa personne, mais également de réaffirmer un pouvoir central vacillant sur les territoires de l'Imerina. Vouées à l'échec, elles initient néanmoins des formes militaro-policières de domination, et de contrôle des populations, reprises par le colonisateur, comme l'institution du fokon'olona et les mpiadidy. Cette analyse tente d'éclairer cette continuité des formes de contrôle. Deuxièmement, elle explicite les impératifs inhérents aux modalités de domination du nouvel arrivant qui s'incarnent dans l'imposition violente de l'Ordre colonial et dans la structuration empirique des forces de maintien de l'ordre. Entre transpositions directes de formes de polices métropolitaines, adaptations impériales et créations endogènes à l'État colonial à Madagascar, la mise au jour de la structuration et de l'institutionnalisation des polices ne permet que d'effleurer les réalités des pratiques policières et de l'action des forces de maintien de l'ordre. L'étude des formes de polices coloniales, des modalités de l'ordre et des pratiques policières à Madagascar apparaît une fois de plus comme un chantier en devenir.

Nicolas Courtin

Université de Versailles Saint-Quentin

47, boulevard Vauban

F-78280 Guyancourt nicolascourtin@gmail.com

\section{BIBLIOGRAPHIE}

About, I., Les fondations d'un système national d'identification policière en France (18931914). Anthropométrie, signalements et fichiers, Genèses, 54, 2004, pp. 28-52.

Balandier, G., La situation coloniale: approche théorique, Cahiers internationaux de sociologie, 1951, 11, pp. 44-79.

Berlière, J.-M., Le monde des polices en France, XIX $X^{e} X X^{e}$ siècles, Bruxelles, Complexe, 1996.

Chapus, G.S., Rainilaiarivony, un homme d'État malgache, Paris, éditions Diloutremer, 1953.

Condominas, G., Fokon'olona et collectivités rurales en Imerina, Homme d'Outre-Mer, Paris, 1960.

Deschamps, H., Gallieni Pacificateur, écrits coloniaux de Gallieni, Paris, Presses Universitaires de France, 1949.

Ellis, S., Un complot colonial à Madagascar, l'affaire Rainandriamanpandry, Collection les Afriques, Karthala, éd. Ambozontany, 1990.

Ellis, S., L'insurrection des Menalamba, Une révolte à Madagascar (1895-1898), ASCKarthala-Éd. Ambazontany, 1998.

Esoavelomandroso, M., La province maritime orientale du «Royaume de Madagascar» à la fin du XIX siècle (1882-1895), Tananarive, 1979.

Fremigacci, J., L'administration coloniale: aspects oppressifs, Omaly sy Anio, Tananarive, 1978, 7-8, pp. 209-237.

Fremigacci, J., Insécurité, banditisme et criminalité dans le Nord de Madagascar au début du $\mathrm{XX}^{\mathrm{e}}$ siècle, Omaly sy Anio, 1987, 25-26, pp. 297-320.

Fremiggaci, J., Les chemins de fer de Madagascar (1901-1936): une modernisation manquée, Afrique \& Histoire, Verdier, octobre 2006, 6. 
Harmand, J., Domination et colonisation, Flammarion, Paris, édition de 1919 (1 ${ }^{\text {ère }}$ édition 1910).

Jacob, G., L'Armée et le pouvoir dans le royaume de Madagascar au temps du Premier ministre Rainilaiarivony (1864-1895, Omaly Sy Anio, 1994, 33-36, pp. 381-402.

Jacob, G., La France et Madagascar de 1880 à 1894. Aux origines d'une conquête coloniale, thèse à l'université Paris-IV, 1996.

Le Chartier, H., Pellerin, G., Madagascar, depuis sa découverte jusqu'à nos jours, Bibliothèque instructive, Paris, Jouvet et Cie éditeurs, 1888.

López, L., Policiers, gendarmes et signalement descriptif. Représentations, apprentissages et pratiques d'une nouvelle technique de police judiciaire, en France à la Belle Époque, Crime, Histoire \& Sociétés / Crime, History \& Societies, 2006, 1, 10, pp. 51-76.

Michel, M., La conception de l'État colonial chez Gallieni, Omaly Sy Anio, 1994, 33-36, pp. 589-601.

Moor, J.A., Wesseling, H.L., Imperialism and war: essays on colonial wars in Asia and Africa, Universitaire de Leiden, 1989.

Paillard, Y. G., D'un protectorat fantôme au fantôme d'un protectorat: les métamorphoses du pouvoir à Madagascar de 1885 à1896, Omaly Sy Anio, 1994, 33-36, pp. 559-583.

Rafidison, R., Conquête et pénétration coloniale dans la province de Farafangana, Omaly $S y$ Anio, 1986, 23.

Raison-Jourde, F., Bible et pouvoir à Madagascar, Invention d'une identité chrétienne et construction de l'État, Paris, Karthala, 1991.

Rakoto, I., Recueil des jugements et arrêts rendus par les tribunaux à Madagascar, 18411896, Paris, Éditions L'Harmattan.

Sinclair, G., Williams, C., Home and away, the cross-fertilisation between 'colonial' and 'British' policing, in Globalising British Policing, Ashgate, Royaume-Uni, 2011.

Valensky, C., Le soldat occulté. Les malgaches de l'Armée française, 1884-1920, Paris, L'Harmattan, 1995.

Vidal, H., Le code des 118 articles dans les sources européennes, communication à L'Académie Malgache dans sa séance du 21 mars 1968.

You, A., Madagascar (Histoire, organisation, colonisation), Paris, Berger Levrault, 1905. 